\title{
ACTUATOR FAULT DETECTION IN AUTONOMOUS HELICOPTERS
}

\author{
G. Heredia ${ }^{1}$, Volker Remuß ${ }^{2}$, A. Ollero ${ }^{1}$, R. Mahtani ${ }^{1}$ and Marek Musial ${ }^{2}$
}

\author{
(1) Robotics, Computer Vision and Intelligent Control Group \\ Dept. Ingeniería de Sistemas y Automática, University of Seville \\ Camino de los Descubrimientos s/n, 41092, Seville, Spain \\ \{guiller,aollero,rajesh\}@cartuja.us.esｈttp://www.esi.us.es/GVR \\ (2) Institut für Technische Informatik und Mikroelektronik (TIME) \\ Technical University of Berlin \\ Einsteinufer 17, D-10587 Berlin, Germany \\ \{remuss,musial\}@cs.tu-berlin.de
}

\begin{abstract}
This paper presents an actuator fault detection and diagnosis system for autonomous helicopters. The system has been tested with the MARVIN autonomous helicopter. Fault detection is accomplished by evaluating any significant change in the behaviour of the vehicle with respect to the fault-free behaviour, which is estimated by using an observer. The effectiveness of the proposed approach is demonstrated by means of MARVIN experimental results and simulations. Copyright (C) 2004 IFAC
\end{abstract}

Keywords: Fault detection; fault diagnosis; autonomous helicopters; UAV; actuators.

\section{INTRODUCTION}

Unmanned Aerial Vehicles are increasingly used in many applications in which ground vehicles cannot access to the desired locations due to the characteristics of the terrain and the presence of obstacles. In many cases the use of aerial vehicles is the best way to approach the objective to get information or to deploy instrumentation.

The work described in this paper has been performed in the framework of the COMETS project funded by the IST Programme of the European Union, devoted to the coordination and control of multiple unmanned aerial vehicles. Particularly, both helicopters and airship have been integrated in the COMETS system.

Helicopters have high manoeuvrability and hovering ability. Then, they are well suited to agile target tracking tasks, as well as to inspection and monitoring tasks that require to maintain a position and to obtain detailed views. Furthermore, the vertical take-off and landing of helicopters is very desirable in many applications. Remotely piloted helicopters are inherently unstable and dynamically fast. Even with improved stability augmentation devices, a skilled, experienced pilot is required to control them during flight. Autonomous helicopter control is a challenging task involving a multivariable nonlinear open-loop unstable system with actuator saturations.

Moreover, helicopters do not have the graceful degradation properties of fixed wing aircrafts or airships in case of failures. Thus, a failure in any part of the autonomous helicopter (actuators, sensors, control system, etc) can be catastrophic. If the failure is not detected and accounted for, the helicopter may crash.
Fault Detection and Isolation (FDI) techniques have been widely used in process industry to detect faults in actuators and sensors. If a fault is detected, the structure of the controller can be changed to get the best possible response of the system, or the system can be stopped. FDI techniques have been applied to autonomous vehicles as cars, aircrafts (Napolitano et al., 1999; 2000), fixed-wing (Napolitano et al., 1998) and tilt-rotor UAVs (Rago et al., 1998), and underwater vehicles (Alessandri et al., 1998). However, the authors have not found any published application to autonomous helicopters.

In this paper, a fault detection system for autonomous helicopter actuators is presented. The system is applied to the Marvin autonomous helicopter. This helicopter is described in section 2 . Section 3 introduces the fault detection and isolation approach. The results on the application of these techniques to Marvin are presented in section 4. Sections 5 and 6 are devoted to the conclusions and references.

\section{THE MARVIN HELICOPTER}

The basis of MARVIN is a conventional model helicopter. It has a rotor diameter of $1.9 \mathrm{~m}$ and is equipped with a two-stroke petrol engine producing about $2 \mathrm{~kW}$. Its takeoff weight amounts to about 12 $\mathrm{kg}$, operation time is approximately 30 minutes. Figure 1 shows a view of MARVIN in flight. The sensors on board of MARVIN are:

1. A custom-built inertial measurement unit consisting of three magnetometers, three semiconductor accelerometers, and three piezoelectric gyroscopes.

2. A fire sensor that detects a certain range of ultraviolet light characteristic of burning wood, 
gas, or oil.

3. Temperature sensor to measure the heat of surrounding air.

4. A light barrier rpm sensor for measuring the main rotor rpm.

5. An ultrasonic rangefinder looking down.

6. A high resolution digital still camera.

7. A NovAtel RT-2 carrier phase differential GPS receiver.

On-board data processing is divided between a PCarchitecture single-board computer (by Compulab) and an Infineon SAB80C167 microcontroller. The $\mathrm{PC}$ is responsible for wireless network communication and for the interfacing of the on-board camera, while the microcontroller is used for sensor and actuator data processing and the implementation of the flight controller.

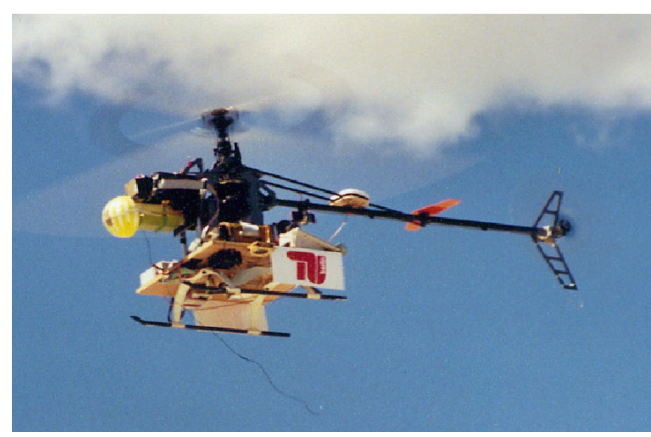

Fig. 1. MARVIN in flight.

MARVIN's actuators are six servos that operate the engine throttle, the tail rotor pitch, and the main rotor pitch settings. The swashplate is moved by four servos, which control its position and orientation at one of four corners with $90^{\circ}$ offset. This means that three parameters (collective pitch $\mathrm{p}_{\mathrm{c}}$, cyclic pitch $\mathrm{p}_{\mathrm{y}}$ for pitching, and cyclic pitch $\mathrm{p}_{\mathrm{x}}$ for rolling) are redundantly affected by four servo outputs, the front servo $s_{f}$, the rear servo $s_{b}$, the left servo $s_{l}$, and the right servo $s_{\mathrm{r}}$. The involved relations are:

$$
\begin{aligned}
& p_{c}=\frac{1}{4}\left(s_{f}+s_{b}+s_{l}+s_{r}\right) \\
& p_{x}=s_{l}-s_{r} \\
& p_{y}=s_{b}-s_{f}
\end{aligned}
$$

The servo signals have to be calculated by the controller according to the above relations. Consequently, if one of the servos fails but can be moved by the remaining three, MARVIN can still operate safely without any special measures. Thus, early fault detection and identification is relevant to increase helicopter safety.

\section{FAULT DETECTION AND ISOLATION}

Safety and reliability are important requirements in man-made dynamical systems. These requirements apply specially to safety-critical systems, as is the case of helicopters. The early detection of faults can help to avoid system shut-down, breakdown and even catastrophes involving human and material damage.

The monitoring of faults in feedback control system components is known as fault detection and isolation (FDI). The procedure of generating a control action which has a low dependency on the presence of certain faults is known as fault tolerant control.

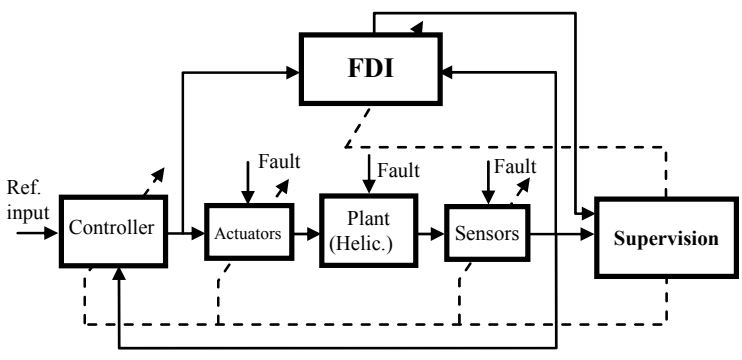

Fig. 2. Scheme of fault tolerant control system with supervision subsystem.

Fig. 2 shows the general schematic arrangement appropriate to many fault tolerant control systems (Patton, 1997) with four main components: the plant itself (including sensors and actuators), the FDI unit, the feedback controller and the supervision system. The plant is considered to have potential faults in sensors, actuators or other components. The FDI unit provides the supervision system with information about the onset, location and severity of any fault. Based on system inputs and outputs together with fault decision information from the FDI unit, the supervision system will reconfigure the sensor set and/or actuators to isolate the faults, and tune or adapt the controller to accommodate the fault effects.

An example of control reconfiguration in autonomous helicopters is RPM control reconfiguration (see Kannan et al., 1999 and Yavrucuk and Prasad, 1999).

In helicopters, the primary method used to control altitude is to change the main rotor collective pitch angle which controls the magnitude of the thrust vector. Normally, the RPM of the main rotor is kept constant using a governor. In case the main rotor collective actuator fails or gets stuck, the amount of thrust may still be controlled by varying the speed (RPM) of the rotor. There is a performance degradation, but altitude can still be controlled using RPM control.

A scenario where such control may be used in practice is during descent where the helicopter needs to increase thrust as it nears the ground. If the main rotor collective actuator fails during this phase of flight, the helicopter will be unable to reduce its descent rate as it gets closer to the ground.

The FDI unit is a basic element of the fault tolerant control scheme presented in Fig. 2. The work presented in this paper concentrates on FDI.

Fault-detection approaches can be classified as model-free and model-based paradigms (Gertler, 1988; Frank, 1990). Model-free fault diagnosis includes all the techniques that do not rely upon models of the underlying system, while model-based methods try to diagnose faults using the redundancy of some mathematical description of the dynamics.

Examples of model-free techniques are the methods 
based on spectral analysis, pattern recognition and statistical classification, and the classical limit and trend check (Pau, 1981).

From the beginning of the seventies, there have been numerous theoretical advancements in fault diagnostics based on analytical redundancy. According to this approach, all the information on the system can be used to monitor the behaviour of the plant, including the knowledge about the dynamics. The presence of faults is detected by means of the so-called residuals, i.e., quantities that are over-sensitive to the malfunctions. Residual generation can be performed in different ways: parity equations (Gertler, 1997), observer-based generation (Patton and Chen, 1997), and the methods based on parameter estimation (Isermann, 1984). Neural networks and fuzzy systems have also been applied in model-based FDI (Patton et al., 2000).

Observer-based and parameter estimation methods are the most frequently applied methods for fault detection (Isermann and Ballé, 1997). Most published work in recent years on FDI systems for autonomous vehicles also use observer-based methods. The basic idea behind the observer or filterbased approach is to estimate the outputs of the system from the measurements by using either Luenberger observer(s) in a deterministic setting or Kalman filter(s) in a stochastic setting.

Several methods have been used for observer generation in autonomous vehicles FDI: Luenberger observers, Kalman filters, banks of observers and Kalman filters and neural networks.

Neural networks have been used to detect sensor and actuator faults applied to a B-747 mathematical model (Napolitano et al., 2000). A bank of Kalman filters and neural networks have also been used for sensor fault detection on a NASA high altitude UAV simulation model (Napolitano et al., 1998), and on B-373 actual flight data (Napolitano et al., 1999). A bank of Kalman filters have been used for fault detection in aircrafts, with application to a linear simulation model of an Eagle-Eye tilt-rotor UAV (Rago et al., 1998). Actuator fault detection in unmanned underwater vehicles has been done using a bank of Kalman filter estimators (Alessandri et al., 1998).

In this paper, the diagnosis of actuator faults in an autonomous helicopter is investigated and the design of a fault detection system is considered, using a model-based approach, with observer-based residual generation, using Luenberger observers.

\subsection{Fault identification scheme.}

The scheme used for fault identification is presented in Fig. 3. $\mathbf{u}$ and $\mathbf{y}$ are the inputs and outputs vectors of the autonomous helicopter, respectively. The observer block is an input-output model of the helicopter in nominal fault-free conditions, obtained by system identification. The output of the observer is an estimation of the output of the helicopter, $\mathbf{y}_{\text {est }}$.
The residuals $\mathbf{R}$ are generated comparing the estimated outputs of the helicopter with the actual outputs. If a fault is present in the system, the fault diagnosis system will analyse the generated residuals to detect and isolate the fault.

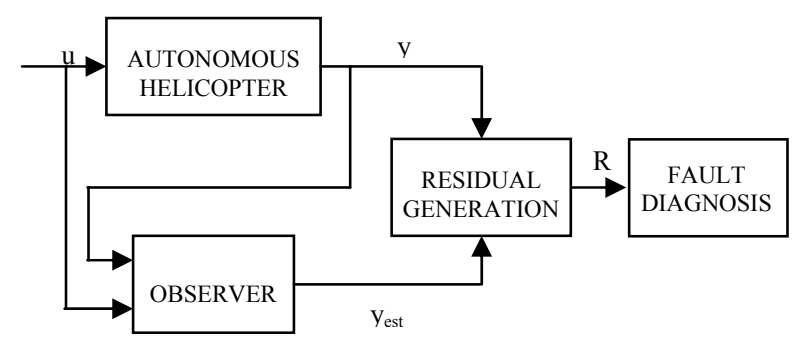

Fig. 3. Fault Identification Scheme.

\subsection{FDI in the MARVIN case}

As the MARVIN helicopter does not have a separate collective pitch actuator, the situation is a little more complicated in practice here. As stated in section 2, if one of MARVIN's main rotor servos gets stuck, both collective and cyclic pitch angles are affected.

Servo failures are frequently located on the internal reduction gears, which cause that the servo can no longer move in one or both directions. In both cases, the failure can be considered as a stuck servo. Other servo failures can be due to electrical problems, and the servo may be unusable, but not stuck, thus letting the other servos to work. The failure can also be located on the mechanical links. So, several different failure types are possible:

- One servo involved in rolling (or pitching) motion has a failure, but does not get stuck. In this case, the collective may also work powered by the other three servos, but the cyclic may not work with only one servo, or be seriously affected.. This situation can be detected as a rolling (or pitching) cyclic actuator fault. Then, the signals to apply the corresponding rolling (or pitching) cyclic command has to be doubled so that the one remaining working servo can move the swashplate to the intended orientation.

- If the servo involved in rolling (or pitching) motion actually gets stuck, both the collective and the rolling (or pitching) actuators will not work. This situation can be detected as a collective actuator fault and a rolling (or pitching) cyclic actuator fault. Provided that the failing servo is not in a state close to a border of its working range (in which case cyclic pitch control would invariably fail), rpm control can be used to govern the lifting force.

- Another less common possibility is that the collective actuator can no longer work, or it may work with a limited range (actually preventing the helicopter from descending, for example), due to a failure in the mechanical links. In this case, a collective actuator fault will be detected. Rpm control can also be used to govern the lifting force.

In the last two cases, if rpm control is applied the cyclic pitch controllers have to consider the rpm 
changes and the error integrator of the controller in question will eventually shift its operating point such that stable and reliable control of the helicopter is regained.

\subsection{Observer design.}

For FDI purposes, only the output estimation is required (Patton and Chen, 1997). The estimation of the state vector is unnecessary. A residual generator based on a Luenberger observer can be used.

As only the outputs are required for FDI, an inputoutput model of the helicopter system can be identified for output prediction. Since past inputs and outputs are available to the FDI system at any given instant, the input-output model can be used to estimate the actual output in fault-free conditions. A linear mathematical model of the input-output links can be used for local analysis. This model can be obtained by means of well known identification schemes. When the signal to noise ratios are high Auto Regressive eXogenous (ARX) models can be used. The ARX model is chosen with the structure that achieve the smallest Akaike's Information Theoretic Criterion (AIC) (Ljung, 1999), according to a simple search algorithm, in which the first half of data is used for estimation and the second for cross validation.
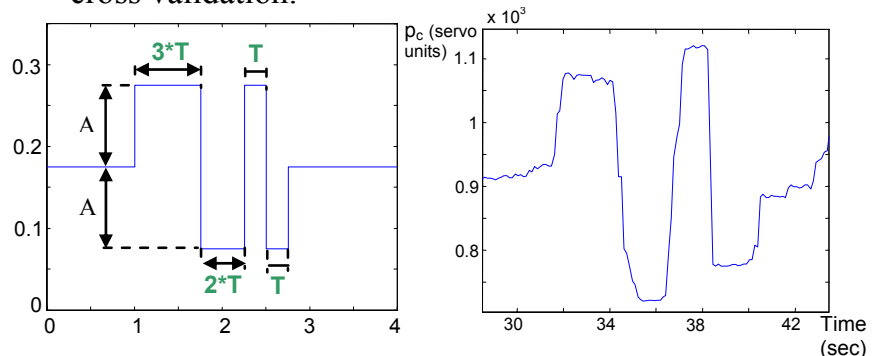

Fig. 4. a) 3211 input signal definition. b) 3211 real collective input signal in a sample experiment.

Input-output data for helicopter output model.

Several experiments have been done to collect MARVIN input-output data for ARX input-output model identification. Input data are the 4 pilot inputs (collective, rolling and pitching cyclics and tail rotor collective). Outputs are helicopter linear and angular velocities, position and inclination angles.

The identification is made on the time domain, and therefore 3211-signals have been used for input excitation. 3211 signals can be seen in Fig. 4. The amplitude $\mathrm{A}$ and the period $\mathrm{T}$ have to be chosen for each experiment, depending on actuator range and the dynamics range that needs to be excited.

In aircraft and helicopter system identification, 3211 signals are preferred to others like steps or doublets, because they excite a broader dynamic spectrum of the system, and also 3211 signals are easy for pilots to execute.

\subsection{Residual generation.}

Independent residuals are constructed for each different actuator failure. Residuals are designed so that they respond to an individual failure and not to the others. In general, residuals $R_{k}$ are functions of the squared difference between estimated and real helicopter linear $\left(v_{i}\right)$ and angular $\left(\omega_{j}\right)$ velocities:

$$
R_{k}=\sum_{i=1}^{3} m_{i}\left(v_{i}-\hat{v}_{i}\right)^{2}+\sum_{j=1}^{3} n_{j}\left(\omega_{i}-\hat{\omega}_{i}\right)^{2}
$$

where $m_{i}$ and $n_{j}$ are weighting coefficients that are determined for each failure based on experience.

\subsection{Residual evaluation.}

Fault diagnosis involves the analysis of the residuals. Several fault diagnosis methods have been reported in the literature (Isermann, 1997). Most of them are in two categories: classification methods (statistical or geometrical), and reasoning methods, that can be used in technical processes where the basic relationships between faults and symptoms (residuals) are at least partially known. This a-priori knowledge can be of help in constructing the residuals, and can be represented in causal relations symptoms $->$ faults. To perform a diagnosis, this qualitative knowledge can be expressed in form of simple rules:

$$
\text { IF < condition }>\text { THEN }<\text { conclusion }>
$$

In autonomous helicopter FDI, this leads to rules as:

$$
\text { IF }<\mathrm{S}_{\text {tail }}>\text { THEN }<\mathrm{F}_{\text {tail }}>
$$

Where $\mathrm{S}_{\text {tail }}$ is true if the tail rotor failure residual goes above the threshold level, and false otherwise. $\mathrm{F}_{\text {tail }}$ denotes that a failure has been detected in the tail rotor actuator. This way, the fault diagnosis system can be used to detect also individual servo failures as mentioned in section 3.2. If a servo involved in rolling motion gets stuck, it will affect simultaneously to two different actuators, collective and rolling cyclic. Then, the corresponding rule is:

$$
\text { IF }<\mathrm{S}_{\text {coll }} \text { AND } \mathrm{S}_{\text {roll }}>\text { THEN }<\mathrm{F}_{\text {rollservo }}>
$$

where $S_{\text {coll }}$ and $S_{\text {roll }}$ are signals that are true if the collective and rolling cyclic failure residuals go above their respective threshold levels, and false otherwise. $F_{\text {rollservo denotes that a failure has been }}$ detected in one of the servos actually involved in the rolling motion.

The first time the residual goes above the threshold level, the fault is supposed to be present, even if the residual goes below it later.

\section{FAULT DETECTION RESULTS}

The actuator fault detection system has been tested using helicopter data from two different sources:

1. A full nonlinear mathematical model, with rigid body dynamics, actuator dynamics, and force and moment generation dynamics, including the flybar. This model is similar to the one presented by Kim and Tilbury, (1998). The model parameters have been identified with MARVIN.

2. Real flight data obtained from MARVIN.

The use of the nonlinear model makes easier to test the actuator fault detection system, because datasets can be easily generated by computer simulation as needed. On the other hand, real flight experiments are the best way of testing the fault detection system, 
although it is more expensive and time consuming, and they are not available at any time.

\subsection{Fault detection using simulated helicopter data.} Using flight data obtained by simulation of the full nonlinear model, the actuator fault detection system has been used to detect faults in all four helicopter actuators: main rotor collective, tail rotor collective and both rolling and pitching cyclic inputs.
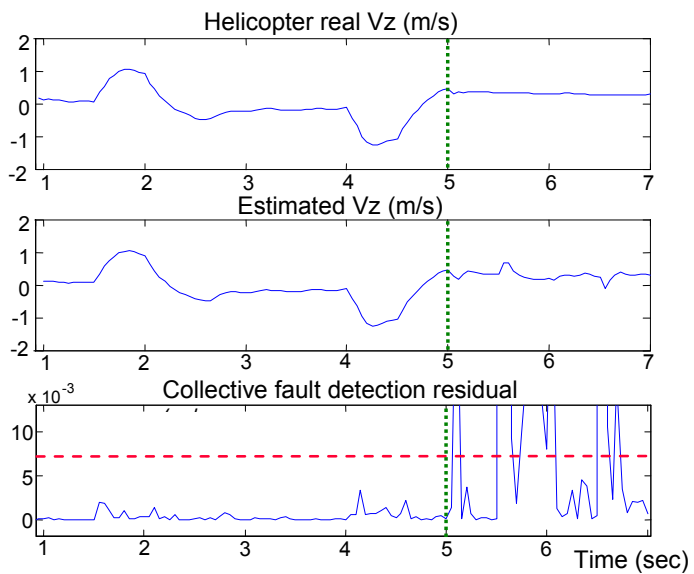

Fig. 5. Collective fault detection (nonlinear model).

In Fig. 5 the results of a collective actuator fault detection is shown. The three plots show the helicopter vertical linear velocity Vz, the Vz estimated by the observer and the evolution of the residual $\left(R=\left(v_{z}-\hat{v}_{z}\right)^{2}\right)$, respectively. At $\mathrm{t}=5 \mathrm{~s}$, the collective actuator gets stuck near the trim hovering value. It can be seen that as a result of the collective failure, the helicopter $\mathrm{Vz}$ remains almost constant while the $\mathrm{Vz}$ estimated by the observer varies. This is captured by the residual evolution, which is below the threshold level for fault-free operation but exceeds the threshold value (dashed line) after the actuator gets stuck, and therefore the collective fault can be detected.

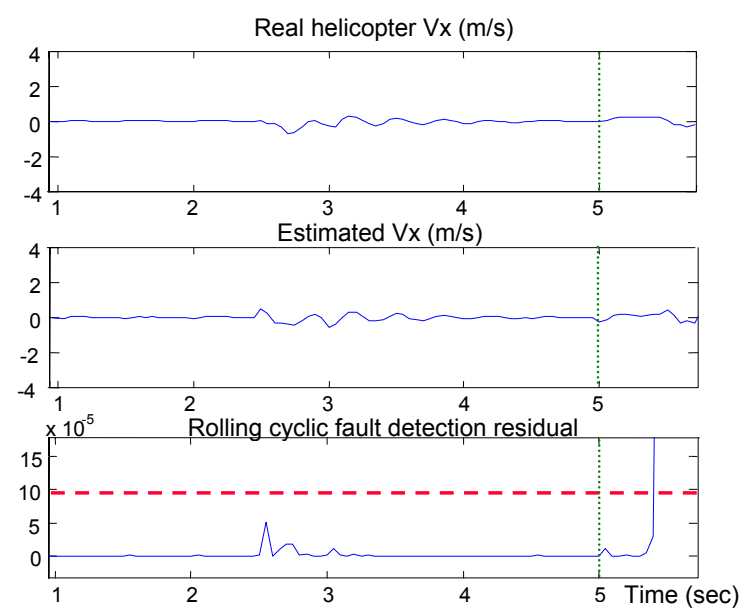

Fig. 6. Rolling cyclic fault detection (nonlinear model).

Fault detection of the rolling cyclic actuator is presented in Fig. 6. The three plots show the real helicopter linear velocity $\mathrm{Vx}$, the $\mathrm{Vx}$ estimated by the observer and the evolution of the rolling cyclic residual $\left(R=\left(v_{x}-\hat{v}_{x}\right)^{2}\right)$, respectively. At $\mathrm{t}=5 \mathrm{~s}$., the rolling cyclic actuator gets stuck near the trim hovering value. In this case, the detection time is larger, but this is due to that the cyclic input is not excited. In fact, it is impossible to detect an actuator failure if the actuator is not excited. In this experiment, the rolling cyclic actuator is not immediately excited, but after a few tenths of a second. Once the cyclic input is excited, the residual goes above the threshold value, and the fault can be detected.

\subsection{Fault detection using real MARVIN flight helicopter data.}

A failure in an actuator can be potentially dangerous for the helicopter, because it can take the helicopter out of control and it may crash. Even for experienced pilots, it could be dangerous to make flight experiments with a faulty actuator.

In order to test the FDI system of the autonomous helicopter in real flight conditions, some experiments were planned to simulate a faulty condition in an actuator while maintaining security of people and the helicopter.
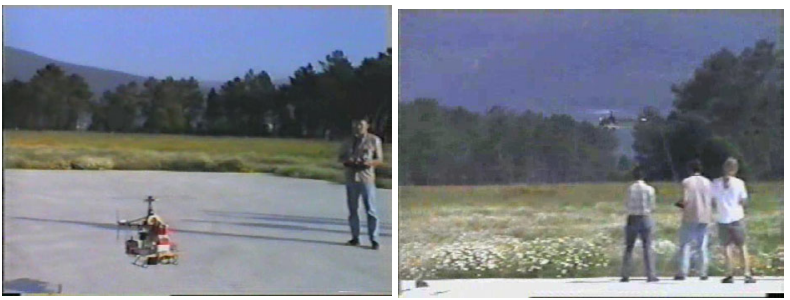

Fig. 7. MARVIN fault detection experiments.

In these experiments, the pilot was commanded to make a flight with different movements, but at a given time, he should maintain one of the actuators almost fixed in a given position, and thus simulating a stuck actuator. These experiments were repeated in different flight conditions (near hover, forward flight at different velocities, etc). Fig. 7 shows a photograph of the experiments. The video sequences can be seen in the COMETS web site (http://www.comets-uavs.org).

After the experiments, the input data was modified. The input signal corresponding to the "stuck" actuator was changed, and a different input command was introduced for that actuator. The effect of this modification is that a varying input signal is commanded to the helicopter, but, because of the stuck actuator, the actuator remains in a fixed position, and all the sensor data correspond to this stuck situation. This is an effective and safe way of reproducing faulty conditions in helicopter actuators.

In Fig. 8 an example actuator fault experiment is presented. A stuck type fault has been reproduced in the main rotor collective of the MARVIN helicopter, using real flight experiment data. At $\mathrm{t}=18 \mathrm{~s}$. (dashed line) the collective actuator gets stuck. Then the collective input commanded by the controller cannot be followed by the actuator, which remains stuck (effective input line in Fig. 8). 


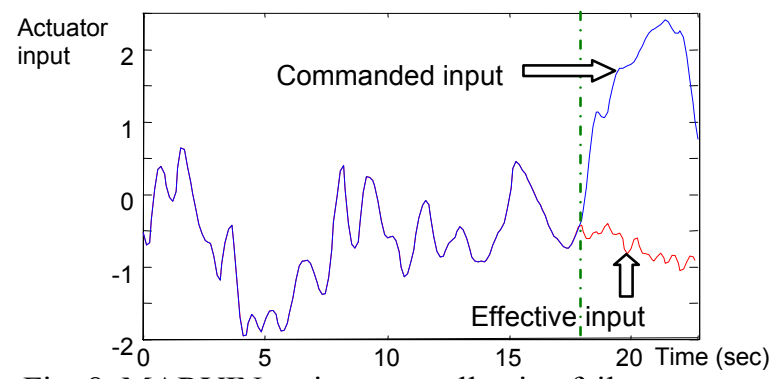

Fig. 8. MARVIN main rotor collective failure.

The collective residual generated by the FDI system can be seen in Fig. 9. It can be seen that the residual is below the threshold level (horizontal dashed line) for normal fault-free operation. But, when the actuator gets stuck (vertical dashed line), the residual goes above the threshold level, and therefore, a collective actuator fault has been detected.

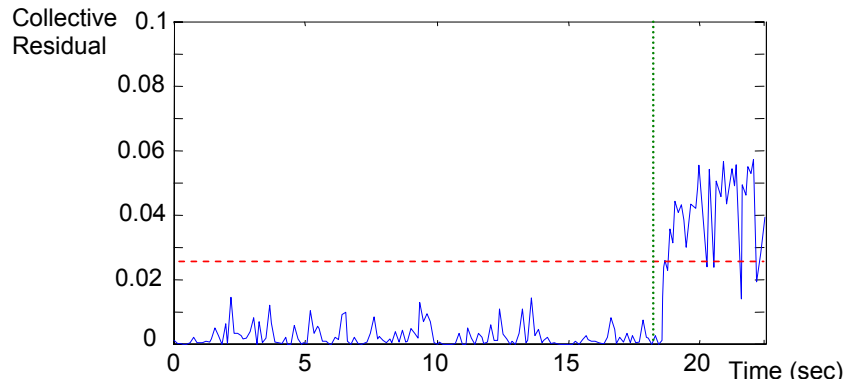

Fig. 9. Time evolution of the collective residual.

\section{CONCLUSIONS}

The application of autonomous helicopters in civilian application requires the improvement of safety conditions to avoid potential accidents. Fault detection and Isolation plays an important role in this context. This paper has presented a system for helicopter's actuator fault detection and its application to the MARVIN autonomous helicopter. The system has been designed by using a full nonlinear model of the helicopter identified by using experimental data. Experiments with MARVIN have been conducted to collect input-output data in many different conditions. Furthermore, the paper presents a validation experiment by reproducing the main rotor actuator failure.

\section{REFERENCES}

Alessandri, A., M. Caccia and G. Veruggio (1999). Fault detection of actuator faults in unmanned underwater vehicles. Control Engineering Practice, 7, pp. 357-368.

Frank, P.M. (1990). Fault diagnosis in dynamic systems using analytical and knowledge-based redundancy. A survey and some new results. Automatica 26(3), 459-474

Gertler, J. (1988). Survey of model-based failure detection and isolation in complex plants. IEEE Control Systems Mag. 9, 3-11.

Gertler, J. (1997). Fault detection and isolation using parity relations. Control Engineering Practice,
5, pp. 653-661.

Isermann, R. (1984). Process fault detection based on modelling and estimation methods-a survey. Automatica, 20(4), 387-404.

Isermann, R. (1997). Supervision, fault-detection and fault-diagnosis methods - An introduction. Control Engineering Practice, 5, pp. 639-652.

Isermann, R. and P. Ballé (1997). Trends in the application of model-based fault detection and diagnosis of technical processes. Control Engineering Practice, 5, pp. 709-719.

Kannan S., J. V. R. Prasad, D. P. Schrage, I. Yavrucuk, L. Wills, and C. Restrepo (1999). Simulation and Flight Control Integration using the Open Control Platform for Unmanned Aerial Vehicles. In AIAA Digital Avionics Conference.

Kim S. and D. Tilbury (1998). Mathematical Modelling and Experimental Identification of a Model Helicopter. Proceedings of the AIAA Modelling and Simulation Technologies Conference, paper AIAA-98-4357, pp 203-213.

Ljung, L. (1999). System Identification - Theory for the User. Prentice-Hall, N.J., 2nd edition.

Napolitano M., D. Windon, J. Casanova, M. Innocenti and G. Silvestri (1998). Kalman filters and neural-network schemes for sensor validation in flight control systems. IEEE Trans. On Control Systems Technology, 6, 596-611.

Napolitano M., Y. An, B. Seanor, S. Pispistos and D. Martinelli (1999). Application of a neural sensor validation scheme to actual Boeing B737 flight data. Proc. Of the '99 AIAA Guidance, Navigation and Control Conference.

Napolitano M., Y. An and B. Seanor (2000). A fault tolerant flight control system for sensor and actuator failures using neural networks. Aircraft Design, 3, 103-128.

Patton, R.J. (1997), Fault-tolerant control systems: the 1997 situation. IFAC Symposium on Fault Detection Supervision and Safety for Technical Processes, Kingston, UK, 3, pp 1033-1054.

Patton, R.J. and J. Chen. (1997). Observer-based fault detection and isolation: robustness and applications. Control Engineering Practice, 5, pp. 671-682.

Patton, R.J., F J Uppal and C J Lopez-Toribio, (2000). Soft Computing Approaches To Fault Diagnosis For Dynamic Systems: A Survey. 4th IFAC Symposium on Fault Detection Supervision and Safety for Technical Processes, Budapest, 14-16 June 2000, 1, pp 298-311.

Pau, L.F. (1981) Failure diagnosis and performance monitoring. Marcel Dekker, New York.

Rago, C., R. Prasanth, R.K. Mehra and R. Fortenbaugh. (1998). Failure detection and identification and fault tolerant control using the IMM-KF with applications to the Eagle-Eye UAV. Proc. Of the $37^{\text {th }}$ Conf. On Decision and Control, Tampa, Florida, USA.

Yavrucuk, I. and J. V. R. Prasad (1999). Simulation of reconfigurable heli-uavs using main rotor rpm control in failure modes. In AIAA Modelling and Simulation Technologies Conference. 